\title{
THEMATIC PAPER \\ Advances in the treatment of substance use disorder in Cyprus
}

\author{
Lampros Samartzis $^{1,2}$
}

${ }^{1}$ Consultant psychiatrist,

Department of Addiction

Psychiatry, Athalassa Hospital,

Mental Health Services, Nicosia,

Cyprus; emai

Cyprus; email:
zis@gmail.com

zis@gmail.com

${ }^{2}$ Psychiatrist, special scientist and clinical collaborator in psychiatry,

Medical School, University of

Cyprus, Cyprus

Conflicts of interest. None.

doi:10.1192/bji.2018.16

(c) The Author 2018. This is an Open Access article, distributed under the terms of the Creative Commons Attribution-

NonCommercial-NoDerivatives Com ativecommons org/licenses/by-nc-nd/4.0/), which permits non-commercial re-use, distribution, and reproduction in any medium, provided the original work is unaltered and is properly cited. The written permission of Cambridge Univer mission of Cambridge University mercial re-use or in order to create a derivative work.
Addiction psychiatry is a relatively new field in Cyprus. This paper presents the advances in the treatment of substance use disorders in the country in the past three decades. These advances have included increased availability of services, increased accessibility, the development of a modern biopsychosocial harm-reduction approach and evidence-based pharmacological treatments.

Cyprus is a member state of the European Union (EU) with a population of 855000 inhabitants, comparably high living standards and a low unemployment rate. Despite its relative wealth, mental healthcare expenditure is considered low compared with the average in the EU. People with substance use disorders in Cyprus usually seek treatment in the public sector but can also be treated privately. The country has 11 psychiatrists per 100000 people, compared with an average of 16 per 100000 in the EU (European Commission, 2017). There are currently only two psychiatrists working full time in the department of addiction psychiatry in the public sector.

\section{Heroin and other opioids}

It is estimated there are up to 1200 high-risk opioid users in Cyprus. In 2016, 253 of them were undergoing opioid substitution treatment (OST). The average number of drug-induced deaths in Cyprus is eight per year, of which $90 \%$ are males who use opioids intravenously (European Monitoring Centre for Drugs and Drug Addiction, 2017). Causes of death include both illegal opioids and legal opioids diverted from medical to recreational use, as well as legally prescribed opioids for medical use.

Until 2007, there was no OST available in Cyprus. The only available treatment was within abstinence-based therapeutic communities. In August 2007 the first OST unit (named 'Gefyra' meaning 'The Bridge') opened in Nicosia, the capital city of Cyprus. The unit started with 13 patients in 2017, increased to 32 in 2011 and to 84 patients in 2017. A low-threshold approach was implemented in this harm-reduction intervention in recent years, especially after 2011, because an increasing body of evidence suggested there were generally better treatment outcomes for low treatment-threshold compared with high treatment-threshold designs (Kourounis et al, 2016). The OST program aimed to improve accessibility to treatment and to offer personalised treatment options regarding medication choice and dose titration, as well as flexibility of treatment duration. There is an emphasis on maintenance, harm reduction and retention of low adherence patients.

In recent years the number of OST units in the public sector has increased from one to five (one in each of the main cities of Cyprus). In addition, an OST service is now available in the prison setting. The main medication that is used in all OST programs is a combination of buprenorphine plus naloxone for short-term as well as for long-term/ maintenance treatment, following established guidelines (Taylor et al, 2015). Methadone is only used for short-term in-patient use until the patient is cross-titrated to a buprenorphinenaloxone combination, which increases safety. In the private sector, oxycodone and dihydrocodeine have also been used for OST.

\section{Cannabis}

In Cyprus, cannabis for personal recreational use is illegal. Medical cannabis is strictly controlled by the Ministry of Health and only a few people are approved each year to receive it, mainly in the form of cannabis oil. Currently, there is a draft law under discussion for the regulation of medical cannabis; it includes a provision for medical use in specific circumstances (House of Representatives, 2018). The prevalence of recreational cannabis use is stable, involving less than $5 \%$ of the population. Nevertheless, within that sub-population there has recently been a decrease in the use of herbal cannabis and an increase in synthetic cannabinoids.

Cannabis-induced psychoses are treated in out-patient psychiatric clinics in the public and private sectors. When accompanied by severe behavioural disturbance, patients are treated in a psychiatric hospital, usually under court-ordered, temporary, obligatory admission to hospital. Cannabis users who commence treatment on an out-patient voluntary basis are usually offered only counselling, with the addition of psychiatric care if they present with psychotic symptoms or with other psychiatric comorbidity.

\section{Cocaine and other stimulants}

In 2016, cocaine, 3,4-methylenedioxymethamphetamine (MDMA) and amphetamines were used only by $0.4,0.3$ and $0.1 \%$ of the population aged 15-34 years, respectively. There are no available data regarding stimulants classified as 
new psychoactive substances. Despite their relatively low popularity among young people, those taking recreational stimulants are receiving treatment disproportionally often; they constitute over $15 \%$ of people that are treated for a substance use disorder. It is notable that stimulants comprised three of the five most commonly seized classes of illicit drugs in Cyprus over the past year (European Monitoring Centre for Drugs and Drug Addiction, 2017).

The prevalence of both cocaine and amphetamine misuse in Cyprus peaked in 2009. There has been a consistent decrease each year since then. MDMA is currently rarely taken and its use has steadily decreased, at least since 2006. Despite this, a recent multi-city wastewater study found Limassol to be among the top ten European cities for crystal methamphetamine use, which is a relatively new drug in Cyprus (European Monitoring Centre for Drugs and Drug Addiction, 2018).

There is no dedicated program for the treatment of stimulant use disorder, but detoxification, rehabilitation and relapse prevention are offered in the general addiction psychiatry setting. Most of these patients also present with mental health comorbidities that are treated in an integrative care setting. The young stimulant misusers are referred to a special counselling centre for adolescents in the public sector.

\section{Alcohol}

The first drug and alcohol detoxification and rehabilitation centre, named 'THEMEA', was established in the public sector in 1991 as a division of the Psychiatric Clinic of the General Hospital of Nicosia. Originally, that unit only had four beds and admitted 'alcoholics' as well as people with illegal substance use disorder, who were known at that time as 'drug addicts'.

Alcohol is a very popular legal recreational substance in Cyprus, with the prevalence of alcohol use disorder just below the European average, at around 3\%. There is an increasing prevalence of alcohol use among adolescents in Cyprus. A recent multi-centre study of 15- to 16-year-old school students found that almost all of them had taken recreational alcohol at some time and 70\% reported alcohol use within the past 30 days, compared with a European average that is less than $50 \%$ at that age (European Monitoring Centre for Drugs and Drug Addiction, 2015).

At present, THEMEA is still the only drug and alcohol detoxification and rehabilitation centre in the public sector in Cyprus. It has been developed into a university clinic that offers full in-patient and out-patient detoxification, rehabilitation and relapse prevention services under the care of an experienced multidisciplinary team including addiction psychiatrists, clinical and counselling psychologists, specialist nurses, an occupational therapist and a social worker. Medical students of the three medical schools of Cyprus as well as residents in psychiatry are trained in this clinic during their rotation in the field of addiction psychiatry. The therapeutic program includes devising an individualised treatment plan. A biopsychosocial approach is used, which is divided into a short-term in-patient phase and a longerterm out-patient phase of treatment and relapse prevention. The treatment consists of a combination of pharmacological and non-pharmacological interventions, according to established guidelines (NICE, 2011). Non-pharmacological interventions include counselling, cognitive-behavioural therapy, mindfulness and behavioural techniques, which take place in an individual and/or group context. Since 2011 the therapeutic program is not entirely abstinence oriented; decreased/controlled alcohol consumption may now be a treatment goal for some patients, in line with published evidence (Adamson et al, 2010; Heather et al, 2010). Medications used in the treatment of alcohol and substance use disorders include naltrexone, nalmefene, disulfiram and baclofen.

Most patients have comorbidities - especially other substance use disorders, mood disorders or personality disorders - which are usually treated by the same multidisciplinary team in an integrative care setting (Prodromou et al, 2014).

\section{Tobacco}

Smoking cigarettes is very common in Cyprus, with $25 \%$ the population $(37 \%$ of men and $14 \%$ of women) aged 15 and above being daily smokers. To help those tobacco-dependent people who want to stop smoking, public mental health services currently offer a structured smoking cessation program consisting of nicotine replacement treatment with patches along with a 3-month counselling intervention (Ministry of Health, 2018).

\section{Discussion}

Cyprus' psychiatric community follows scientific evidence and international medical guidelines in the treatment of mental health disorders. Substance use disorders are considered to be chronic remitting and relapsing mental health disorders, as classified in DSM-5 (2013) and the forthcoming ICD-11. Treatment of legal or illegal substance use disorders is offered on a voluntary basis, in the public sector or privately. Despite this, drug possession for personal use is regarded by the law as a serious criminal offence. It is punishable by up to 12 years in prison for class-A drugs (opioids and cocaine). Recently, new legislation allows young drug users who are arrested for the first time to opt for treatment instead of prosecution and imprisonment.

As substance use disorders are usually chronic conditions, patients need long-term care using an evidence-based multidisciplinary biopsychosocial approach. The health services in Cyprus are changing to implement recent laws regarding the economic and administrative autonomy of public hospitals and an emerging general health system. Modern health policies, integrated with 
medical research achievements and clinical guidelines, will play an important role in advancing further the treatment of substance use disorder.

\section{References}

Adamson S. J., Heather N., Morton V., et al (2010) Initial preference for drinking goal in the treatment of alcohol problems: II. Treatment outcomes. Alcohol and Alcoholism, 45, 136-142.

European Commission (2017) Mental Health: How Many Psychiatrists in the EU? Eurostat. Available at: http://ec.europa.eu/eurostat/web/ products-eurostat-news/-/EDN-20171010-1?inheritRedirect=true (accessed 25 March 2018)

European Monitoring Centre for Drugs and Drug Addiction (2015) The European School Survey Project on Alcohol and other Drugs. Available at: http://www.espad.org/report/country-summaries\#cyprus (accessed 25 March 2018).

European Monitoring Centre for Drugs and Drug Addiction (2017) Cyprus Country Drug Report 2017. Available at: http://www.emcdda. europa.eu/publications/country-drug-reports/2017/cyprus_en (accessed 25 March 2018).

European Monitoring Centre for Drugs and Drug Addiction (2018) Wastewater Analysis and Drugs, a European Multi-City Study. Available at: http://www.emcdda.europa.eu/system/files/publications/ 2757/POD_Wastewater\%20analysis.pdf (accessed 25 March 2018).
Heather N., Adamson S. J., Raistrick D., et al (2010) Initial preference for drinking goal in the treatment of alcohol problems: I. Baseline differences between abstinence and non-abstinence groups. Alcohol and Alcoholism, 45, 128-135.

House of Representatives, Health Committee, Pending Commission Regulations (2018) Available at: http://www2.parliament.cy/ parliamentgr/008_07.htm (accessed 25 March 2018).

Kourounis G., Richards B. D. W., Kyprianou E., et al (2016) Opioid substitution therapy: lowering the treatment thresholds. Drug and Alcohol Dependence, 161, 1-8.

Ministry of Health, Cyprus (2018) Smoking Cessation Programs. Ministry of Health. Available at: https://www.moh.gov.cy/Moh/MOH. nsf/All/4DE89017D625FCF042257997003A4C42?OpenDocument (accessed 25 March 2018).

National Institute for Health and Care Excellence (NICE) (2011) Alcohol-Use Disorders: Diagnosis, Assessment and Management of Harmful Drinking and Alcohol Dependence. NICE. Available at: https:// www.nice.org.uk/guidance/cg115 (accessed 25 March 2018).

Prodromou M., Kyritsi E. \& Samartzis L. (2014) Dual diagnosis affects prognosis in patients with drug dependence in integrative care setting. Health Science Journal, 8, 216-228.

Taylor D., Paton C. \& Kapur S. (2015) The Maudsley Prescribing Guidelines in Psychiatry. John Wiley \& Sons.

\title{
MENTAL HEALTH LAW PROFILES \\ Mental health law in the Former Yugoslav Republic of Macedonia
}

\author{
Antoni Novotni, ${ }^{1}$ Nensi Manusheva ${ }^{1}$ and Gabriela Novotni ${ }^{2}$
}

${ }^{1}$ University Psychiatry Clinic, Skopje, Former Yugoslav Republic of Macedonia; email prof. novotni@gmail.com

${ }^{2}$ University Neurology Clinic, Skopje, Former Yugoslav Republic of Macedonia

\section{Conflict of interest: None.}

doi:10.1192/bji.2017.18

(C) The Authors 2018. This is an Open Access article, distributed under the terms of the Creative Commons Attribution-

NonCommerical-NoDerivatives licence (http://creativecommons. org/licenses/by-nc-nd/4.0/), which permits non-commercial re-use distribution, and reproduction in any medium, provided the original work is unaltered and is properly cited. The written permission of Cambridge University Press must be obtained for com Press must be obtained for commercial re use or in order to create a derivative work.
Psychiatrists are often confronted with the problem of non-consensual treatment. This paper focuses on the rights of patients with mental health disorders in the Former Yugoslav Republic of Macedonia in relation to non-consensual treatment and deprivation of liberty. The current mental health legislation and its implementation in local services is described with an emphasis on the assessment and treatment of patients with mental disorders.

\section{Mental health reforms in Former Yugoslav Republic of Macedonia}

Following its declaration of independence in 1991, the Former Yugoslav Republic (FYR) of Macedonia has adopted an extensive set of legal reforms. Several international legislative documents have been signed and ratified including the Convention Against Torture and Other Cruel, Inhuman or Degrading Treatment or Punishment (Law on Mental Health, 2006).

The FYR of Macedonia Parliament signed up to the National Mental Health Policy on 13
October 2005 (Law on Mental Health, 2006). The Policy includes the following components: developing community mental health services, downsizing large mental health hospitals, developing a mental health component in primary healthcare, development of human resources, involvement of patients and families, advocacy and promotion, human rights protection of patients, equity of access to mental health services across different groups, financing, quality improvement and monitoring systems.

This Policy also addresses and regulates issues such as access to mental healthcare, including the access to the least-restrictive care; rights of mental health service consumers, family members and caregivers; competency, capacity and guardianship issues for people with mental illness; voluntary and involuntary treatment; accreditation of facilities; law enforcement and other judicial system issues for people with mental illness; and mechanisms aimed at overseeing involuntary admission and associated treatment practices. However, there are still no mechanisms enabling the implementation of this Policy and, in practice, the implementation of new guidelines has been very slow.

Regular inspections and complaints processes are reviewed by a national human rights review 